\title{
Characterisation of the main belt asteroid (223) Rosa A proposed flyby target of ESA's JUICE mission
}

\author{
C. Avdellidou ${ }^{1}$, M. Pajola ${ }^{2}$, A. Lucchetti ${ }^{2}$, L. Agostini ${ }^{3,2}$, M. Delbo ${ }^{1}$, E. Mazzotta Epifani ${ }^{4}$, J. Bourdelle de Micas ${ }^{5}$, \\ M. Devogèle ${ }^{6}$, S. Fornasier ${ }^{5,7}$, G. van Belle ${ }^{8}$, N. Bruot ${ }^{1}$, E. Dotto ${ }^{4}$, S. Ieva ${ }^{4}$, G. Cremonese ${ }^{2}$, and P. Palumbo ${ }^{9,10}$ \\ ${ }^{1}$ Université Côte d'Azur, CNRS-Lagrange, Observatoire de la Côte d'Azur, CS 34229, 06304 NICE Cedex 4, France \\ e-mail: chrysa.avdellidou@oca.eu \\ 2 INAF - Astronomical Observatory of Padova, Vicolo Dell'Osservatorio 5, 35122 Padova, Italy \\ 3 Center of Studies and Activities for Space, CISAS, G. Colombo, University of Padova, 35131 Padova, Italy \\ ${ }^{4}$ INAF - Osservatorio Astronomico di Roma, Via Frascati 33, 00040 Monte Porzio Catone, RM, Italy \\ 5 LESIA, Université de Paris, Observatoire de Paris, Université PSL, Sorbonne Université, CNRS, 92190 MEUDON, France \\ 6 Arecibo Observatory, University of Central Florida, HC-3 Box 53995, Arecibo, PR 00612, USA \\ 7 Institut Universitaire de France (IUF), 1 rue Descartes, 75231 PARIS CEDEX 05, France \\ 8 Lowell Observatory, 1400 W. Mars Hill Road, Flagstaff, AZ 86001, USA \\ 9 Dipartimento di Scienze e Tecnologie, Università Parthenope, Centro Direzionale Isola C4, 80143 Napoli, Italy \\ 10 INAF - Istituto di Astrofisica e Planetologia Spaziale, Via Fosso del Cavaliere 100, 00133 Roma, Italy
}

Received 6 November 2021 / Accepted 25 November 2021

\section{ABSTRACT}

\begin{abstract}
Context. The ESA JUICE space mission, on its way to study Jupiter's environment and icy moons, will pass twice through the main asteroid belt. For this reason, the possibility to perform an asteroid flyby has been investigated.

Aims. We aim to gain insight into the physical properties of the outer main belt asteroid (223) Rosa, which has been proposed as a potential JUICE flyby target.

Methods. We report new visible and near-infrared spectroscopic observations at different rotation phases. Additionally, we perform a literature review of all the available physical properties, such as diameter, albedo, mass, and rotational period.

Results. We find that asteroid Rosa is an X-type asteroid that shows no significant spectral variability combining the new and literature spectroscopic data. Its large size and orbital semimajor axis in the outer main belt indicate that Rosa does not belong to the Themis family, while its albedo is only marginally compatible with the family. Rosa's estimated density is in agreement with those of other low-albedo X-type asteroids. Hence, we propose that Rosa is a planetesimal that accreted in the protoplanetary disk beyond the snow line.
\end{abstract}

Key words. minor planets, asteroids: individual: (223) Rosa - techniques: spectroscopic - catalogs - virtual observatory tools

\section{Introduction}

The JUpiter ICy moons Explorer (JUICE) is the first large-class mission of the European Space Agency (ESA) Cosmic Vision 2015-2025 programme; it will investigate Jupiter's atmosphere, magnetosphere, and system of rings. JUICE will mainly focus on Jupiter's giant icy moon Ganymede as a planetary body and potential habitat, characterising its exosphere and ionosphere; the intrinsic magnetic field; the topography, geology, and composition of the icy surface; and its subsurface ocean layers and possible subsurface water reservoirs. Before entering orbit around Ganymede, JUICE will perform multiple close-proximity flybys of Europa and Callisto. Thus, JUICE observations will return an unprecedented comparative picture of Jupiter's icy Galilean Moons (Grasset et al. 2013), detailing the habitability conditions of icy worlds in planetary systems.

During the approximately nine-year cruise phase (see Fig. 1), the spacecraft will perform four Earth flybys and one Venus flyby, and will cross the main asteroid belt twice. The latter is a unique opportunity to fly by and investigate at close range one or more asteroids, hence maximising the scientific return of the full mission as previously demonstrated by the Rosetta closest approach of asteroids (2867) Steins (Keller et al. 2010), and (21) Lutetia (Sierks et al. 2011). To identify an asteroid that could become a possible target of opportunity (ToO) for the JUICE cruise phase (Fig. 1), a dedicated code was developed, called the Automatic Asteroid Search Toolkit, ${ }^{2}{ }^{2}$ ST (for a detailed description, see Agostini et al., in prep.). After retrieving the Spice Kernel Dataset (hereafter SPK, Acton 1996) of the JUICE mission ${ }^{1}$ and the SPK for all size-known asteroids $(\sim 140000)^{2}$, this software determines the minimum distance between the orbit of each asteroid and the JUICE spacecraft. Out of the list of potential objects ordered by range, we used the sizes of the asteroids to calculate the diameter $D$ on the focal plane of the main optical camera of JUICE's payload JANUS (Jovis, Amorum ac Natorum Undique Scrutator; Palumbo et al. 2014). We derived that asteroid (223) Rosa (hereafter Rosa), with $D=82 \mathrm{~km}$, at the closest flyby distance for the current JUICE nominal trajectory $\left(4.290 \times 10^{6} \mathrm{~km}\right.$, occurring on 15 October 2029, Fig. 1) is the only asteroid whose size ( $4 \operatorname{arcsec})$ will marginally exceed the 1 pixel on JANUS, with a resolution of $\sim 3.1$ arcsec $\mathrm{pxl}^{-1}$. The JANUS study of Rosa would entirely

\footnotetext{
1 https://www . cosmos.esa.int/web/spice/

spice-for-juice

2 https://ssd.jpl.nasa.gov/horizons/
} 
focus on multi-band photometry and rotational curve analysis (e.g., Lamy et al. 2010). Nevertheless, if a much closer approach flyby of Rosa could be performed, like the one that Rosetta did with Lutetia at a few thousand kilometres (Schulz et al. 2012), shape reconstruction through stereo acquisitions (Preusker et al. 2012) and resolved spectrophotometry aiming to identify surface variegation (Magrin et al. 2012) could both be performed. Moreover, multiple geomorphological studies would be accomplished, such as the identification of units and their relationship to the stratigraphic record (Massironi et al. 2012), lineament extraction (Thomas et al. 2012), cratering age determination (Sierks et al. 2011), boulder formation and sizefrequency distribution (Küppers et al. 2012), and maximising the scientific return of the JUICE mission.

Rosa is one of the most promising targets; nevertheless, despite its large size, it was previously not well studied. In particular, different spectral classes (indicative of composition) have been assigned to this body. In this work we reassess the diameter, albedo, and mass of Rosa from literature data (Sect. 2). In Sect. 3 we describe our observational effort to spectrally characterise Rosa. In Sect. 4 we discuss in detail the association of Rosa with the nearby Themis asteroid family and the homogeneity of its spectral signature.

\section{Physical properties of (223) Rosa}

Rosa resides in the outer main belt with proper semimajor axis $a_{P}=3.0901 \mathrm{au}$, proper eccentricity $e_{P}=0.1347$, and sine of the proper inclination $\sin \left(i_{P}\right)=0.0266$, while its perihelion $q$ reaches 2.72 au from the $\mathrm{Sun}^{3}$. From the Minor Planet Physical Properties Catalogue ${ }^{4}$, which collects published physical properties of asteroids, we retrieved 12 radiometric diameters for Rosa that were derived using WISE and IRAS data, as well as the respective geometric albedos $p_{V}$. The diameters range between 72 and $109 \mathrm{~km}$, with an uncertainty-weighted average of $D=$ $82.7 \pm 8.4 \mathrm{~km}$, while the albedos range between 0.02 and 0.04 with the uncertainty-weighted average of $p_{V}=0.031 \pm 0.005$ (see Table A.1). In this work (see also Sect. 4) all uncertaintyweighted averages use $1 / \sigma^{2}$ as weights, where $\sigma$ is the error of each measurement. The mass $m$ of Rosa was measured from its perturbations on the orbit of Mars using planetary ephemerides (Table A.1). Folkner et al. (2014) measured a mass that is 1.6 times higher than the later estimation by Fienga et al. (2019); however, the former do not provide error estimates. Using the latest measurement of Rosa's mass by Fienga et al. (2019) and the above-derived average $D$ (where $D$ is taken as the equivalent of a sphere with the same volume as the asteroid) we compute a bulk density to be $\rho=1790 \mathrm{~kg} \mathrm{~m}^{-3}$, with a relative uncertainty of about $50 \%$, which is essentially driven by the uncertainty on the mass determination (Fienga et al. 2019). Finally, the rotation period of the asteroid is $20.283 \mathrm{~h}$ with an amplitude of 0.13 mag with two asymmetric maxima and minima per cycle (Pilcher 2012).

Asteroid spectra in the visible (VIS) and near-infrared (NIR) are used to understand the optical properties and composition of the uppermost part of a body's surface. From the literature we collected all the available spectral and spectrophotometric data for Rosa. We retrieved two VIS spectra (Lazzaro et al. 2004;

\footnotetext{
Orbital elements retrieved from the Minor Planet Center and from the Asteroid Families Portal (http://asteroids.matf.bg.ac.rs/ fam/properelements . php), on October 4, 2021.

4 MP3C, mp3c. oca. eu, developed and maintained at the Observatoire de la Côte d'Azur, is a Service National d'Observation (SNO) of Institut des Sciences de l'Univers (INSU), CNRS.
}
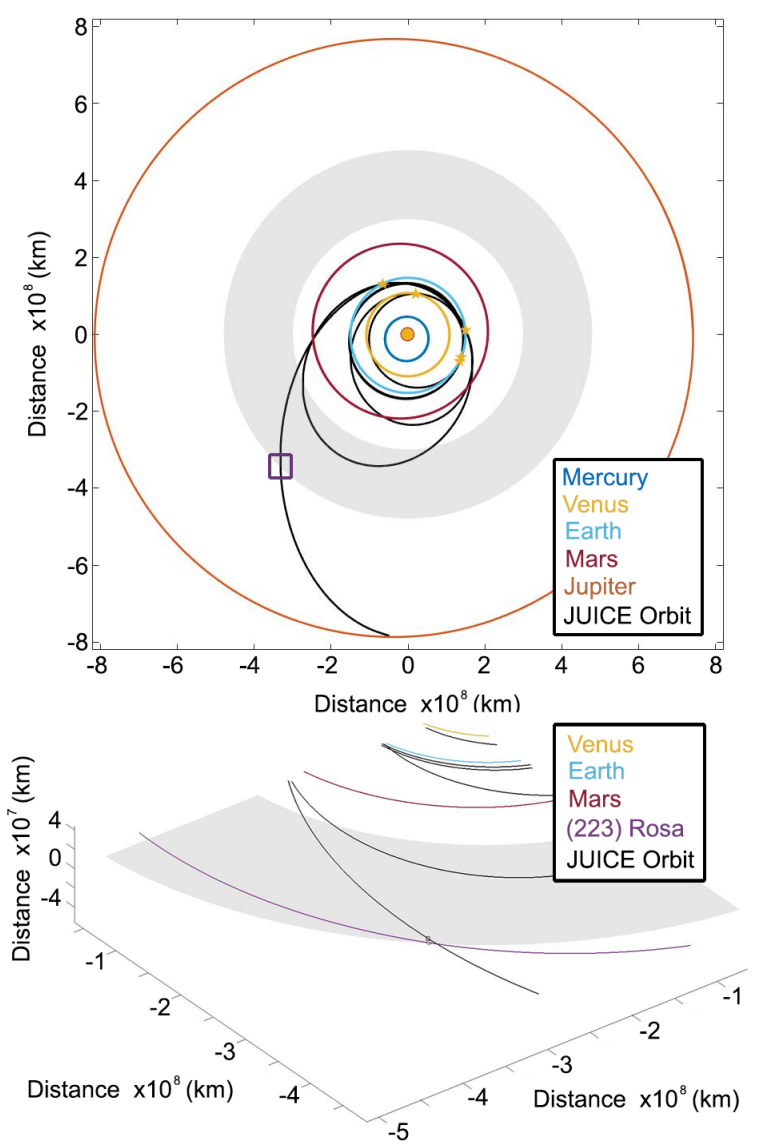

Fig. 1. JUICE trajectory. Top: current baseline orbit of the JUICE mission. The yellow stars show the four Earth gravity assists and one Venus flyby. The grey circular band shows the main asteroid belt extension. The purple box is where the JUICE - Rosa flyby occurs. Bottom: closeup view of the top panel, showing the 15 October 2029 closest approach between the JUICE spacecraft (black curve) and the asteroid Rosa (purple curve).

Fornasier et al. 2011), one NIR spectrum (Marsset et al. 2016) and one set of multi-band spectrophotometry (Zellner et al. 1985). The VIS spectrum by Lazzaro et al. (2004) was classified in the Tholen (Tholen 1989) and Bus (Bus \& Binzel 2002) taxonomies as an X- and Xc-type asteroid, respectively. The VIS spectrum obtained by Fornasier et al. (2011) was classified as a C-type in the Bus taxonomy. The NIR spectrum was classified in the Bus-DeMeo taxonomy (DeMeo et al. 2009) as a Ttype (Marsset et al. 2016). Spectrophotometric data exist in the Eight Color Asteroid Survey (ECAS, Zellner et al. 1985) where Rosa was classified as an X-type in the Tholen taxonomy by Tholen (1989). All literature spectroscopic data are presented in Table A.2.

\section{Spectroscopic characterisation}

Because Rosa was observed during the years at different epochs and phase angles and its classification gave different results (Table A.2), we performed new spectroscopic observations, in the VIS and the NIR wavelength ranges. In addition, Rosa is a large asteroid with a slow rotation, which enables the search for spectral rotational variability because our integration time during the observations is very small compared to its rotational period. For this reason spectra were deliberately taken to cover different phases during a rotation period (see Table 1 and Fig. B.1). 
Table 1. NIR and VIS observations.

\begin{tabular}{lccclllllll}
\hline \hline$N$ & $\begin{array}{c}\text { Date\&Time } \\
(\mathrm{UT})\end{array}$ & $\begin{array}{c}\alpha \\
\left(^{\circ}\right)\end{array}$ & WL & Telescope & Instrument & Airmass & $\begin{array}{l}r \\
(\mathrm{au})\end{array}$ & $\begin{array}{l}\delta \\
(\mathrm{au})\end{array}$ & SA & Local star \\
\hline 1 & $2021-08-21 T 13: 47: 52.0$ & 17.9 & NIR & IRTF & SpeX & 1.03 & 3.07 & 2.55 & SA 93-101 \\
2 & $2021-08-22 T 12: 56: 21.0$ & 17.8 & NIR & IRTF & SpeX & 1.11 & 3.07 & 2.54 & SA 93-101 & $* 64$ Cetus \\
3 & $2021-08-24 T 14: 15: 10.0$ & 17.6 & NIR & IRTF & SpeX & 1.01 & 3.65 & 2.51 & SA 93-101 & \\
4 & $2021-10-02 T 01: 05: 43.4$ & 8.6 & VIS & Copernico & AFOSC & 1.20 & 3.02 & 2.00 & SA 115-271 \\
5 & $2021-10-12 T 23: 54: 36.0$ & 4.7 & NIR & TNG & NICS & 1.16 & 2.97 & 2.03 & SA 115-271 & BD+09445 \\
6 & $2021-10-17 T 10: 16: 18.2$ & 3.0 & VIS & LDT & DeVeny & 1.32 & 2.96 & 2.06 & SA 93-101 & \\
\hline
\end{tabular}

Notes. $\alpha$ is the phase angle at the time of observations (at the beginning of the integration), $r$ the heliocentric range, and $\delta$ the geocentric range. The telescope set-up, use of the SA and local G stars, and data reduction are described in Appendix B.

We normalised all the spectra at the overlapping wavelength of $0.8 \mu \mathrm{m}$. An average spectrum was created by calculating the geometric mean and standard deviation of the reflectance data in 150 bands equally distributed between 0.4 and $2.5 \mu \mathrm{m}$ and with a width of $0.025 \mu \mathrm{m}$ (Fig. 2). This average spectrum was then classified in the Bus-DeMeo taxonomy (DeMeo et al. 2009) by calculating the value of the $\chi^{2}$ figure of merit (Eq. (1)) between the reflectance of the asteroid spectrum $R(\lambda)$ and the average reflectance of each Bus-DeMeo class $R_{M}(\lambda)^{5}$

$\chi_{M}^{2}=\sum_{\lambda} \frac{\left(R(\lambda)-\alpha R_{M}(\lambda)\right)^{2}}{\sigma(\lambda)^{2}}$ with $\alpha=\frac{\sum_{\lambda} R(\lambda) R_{M}(\lambda)}{\sum_{\lambda} R_{M}(\lambda)^{2}}$,

where $\sigma(\lambda)$ is the reflectance uncertainty per wavelength. The two best-matching classes, those with the minimum $\chi^{2}$, are in order $X$ and $T$. Moreover, after visual inspection the $X$ class seems more appropriate due to a better match in the VIS part of the spectrum where the T- and D-type Bus-DeMeo averages deviate. Given the X-type spectral class and very low albedo, Rosa would be a P-type in the Tholen taxonomy.

\section{Discussion}

All the spectra acquired in August and October 2021 lie within $1 \sigma$ of the mean spectrum. Using all our NIR spectra we calculate an average slope of $28.3 \pm 3.41 \% \mu^{-1}$, where the standard deviation of our measurements is smaller than the slope uncertainty of $4.2 \% \mu^{-1}$ that Marsset et al. (2020) derived after correcting the long-term variabilities using SpeX data. When the Italian Telescopio Nazionale Galileo (TNG) spectrum is excluded and only the Infrared Telescope Facility (IRTF) data are averaged, we estimate the spectral slope to be $29.8 \pm 2.45 \% \mu \mathrm{m}^{-1}$, which is consistent with the previous value.

Since these spectra were obtained at very different rotational phases of Rosa (Fig. B.1), according to our spectral characterisation, Rosa seems remarkably homogeneous (Fig. 2). The only different spectrum is that of Marsset et al. (2016), which has a redder slope. If we remove this spectrum from the average and re-compute the best-matching taxonomy, we obtain the same answer (X-type). We also re-classified the Marsset et al. (2016) NIR spectrum in the Bus-DeMeo taxonomy, finding in order that the $\mathrm{D}$ and $\mathrm{X}$ types are the best-matching ones.

Spectral variability implied by the comparison between the Marsset et al. (2016) data and ours cannot be attributed to an aspect angle difference since the viewing geometries were very similar. In particular, using the JPL HORIZONS ephemeris service we calculated the heliocentric ecliptic longitude of our

\footnotetext{
5 http://smass.mit.edu/_documents/

busdemeo-meanspectra.xlsx
}

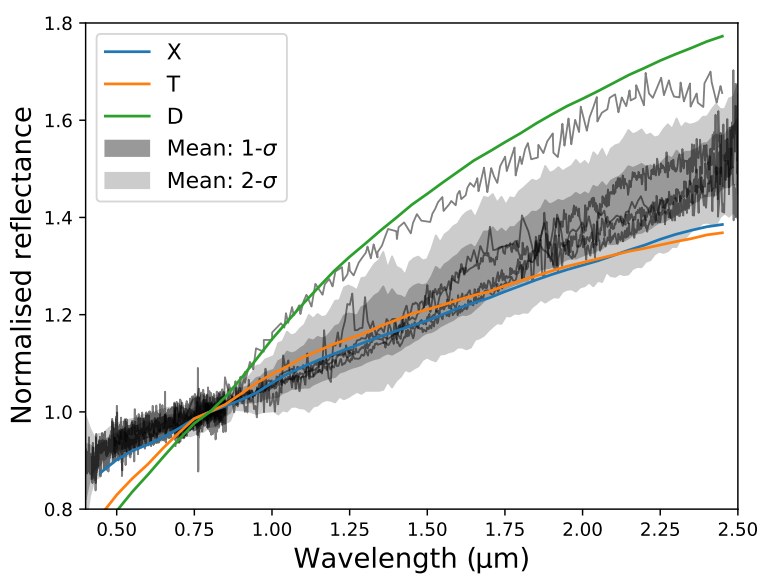

Fig. 2. Reflectance spectra normalised to 1 at $0.8 \mu \mathrm{m}$ (black curves) and their mean spectrum. In dark and light grey are represented the $1 \sigma$ and $2 \sigma$ deviation from the mean spectrum, respectively. For comparison, also shown are the $\mathrm{X}, \mathrm{T}$, and D Bus-DeMeo classes.

observations to be $19.5-20^{\circ}$ at the IRTF and $29^{\circ}$ at the TNG, while the heliocentric ecliptic longitude at the time of the Marsset et al. (2016) observations was at $24^{\circ}$, and thus intermediate between our IRTF and TNG observations. In an extreme case, and accepting the significance of the spectral difference between our NIR data and that of Marsset et al. (2016), we could consider that the redder slope of Marsset et al. (2016) spectrum may be due to a fresher part of the surface, probably caused by an impact event that exposed the non-irradiated subsurface (Fornasier et al. 2007). Unfortunately, the observations by Marsset et al. (2016) were performed 11 years ago and it is impossible to trace back the rotation phase of the asteroid. Thus, currently, we cannot firmly claim asteroid spectral variability on the basis of the current dataset, while JUICE may answer this question.

Currently, asteroid Rosa is considered a member of the Themis asteroid family. Themis is located at the outer main belt and has an age of $2.5 \pm 1$ Gyr (Brož et al. 2013). Its largest object is asteroid (24) Themis, with $D=183.9 \pm 3.3 \mathrm{~km}$ and $p_{V}=0.082 \pm 0.006$, and includes more than 4782 asteroid members (Nesvorny et al. 2015). Rosa is considered the fifth largest object in the family. However, it is understood that asteroid families may include members that do not necessarily share a common origin. These asteroids, which have been linked by traditional family identification methods (e.g., HCM by Zappalà et al. 1990, 1995), may be members of adjacent and overlapping families (Nesvorny et al. 2015; Delbo et al. 2019) or belong to the so-called background population that may consist of currently 
undiscovered asteroid families (Delbo et al. 2017). It is also possib,le that family interlopers such as Rosa are not members of any other nearby family (known or unknown), hence are not collisional fragments; instead, they are original planetesimals (Delbo et al. 2017). These planetesimals have not suffered catastrophic collisions yet (at least in the main belt) in order to produce their own families of fragments, but rather remain intact. This last category of family interlopers may be found among the largest members of families because recent observational and theoretical studies showed that the original planetesimals are expected to be larger than $\sim 50 \mathrm{~km}$ in diameter (Delbo et al. 2017, 2019), as is the case of Rosa. In the following we examine whether Rosa could be a member of Themis or an original planetesimal.

The members of an asteroid family in the $a_{p}$ axis versus $1 / D$ space form a characteristic $\mathrm{V}$ shape, where the centre of the "V" indicates the centre of the family (Vokrouhlický et al. 2006). This occurs because, after the collisional family forming event, the non-gravitational forces due to the Yarkovsky effect cause the asteroid fragments to migrate to smaller or larger heliocentric distances depending on whether they are retrograde or prograde rotators, respectively. The strength of the Yarkovsky effect is inversely proportional to the asteroid diameter $(D)$, resulting in the formation of the $\mathrm{V}$ shape. The opening of the sides of the V also gives the age of the family (Vokrouhlický et al. 2006; Spoto et al. 2015). In the vast majority of cases the family members should reside inside this $\mathrm{V}$ shape.

However, as mentioned before, in the current catalogue of dynamical asteroid families, several families include interlopers that indeed reside well outside of the borders of the $\mathrm{V}$ shapes. This becomes more prominent for asteroids with large diameters, comparable to the family parent body (e.g., Avdellidou et al. 2018). The study of the physical properties of these interloping asteroids may also reveal a different composition, supporting the fact that we should not consider them to be family members. Figure 3 presents the $\mathrm{V}$ shape of the Themis asteroid family with the position of Rosa lying outside of the V.

Since the $\left(a_{p}, 1 / D\right)$ of Rosa already places it outside the Themis family, we investigate whether a different origin is also supported by other physical properties. Using the MP3C database we retrieve albedo values for 2286 Themis family members $(\sim 50 \%)$, and we compute a mean $p_{V}=0.0698$ with a standard deviation of 0.025 ( $1 \sigma$ of the $p_{V}$ distribution), while the uncertainty-weighted average $p_{V}$ of Rosa is $0.031 \pm 0.005$ and thus only marginally consistent $(1.6 \sigma)$ with the Themis family $p_{V}$ distribution, which might be an additional indication that Rosa is not a member of the family. Previous works studied spectroscopically, in the VIS and NIR, members of the Themis family as well as members of the Beagle family (Florczak et al. 1999; Lazzaro et al. 2004; Clark et al. 2010; Fornasier et al. 2011, 2016; de León et al. 2012; Marsset et al. 2016; Kaluna et al. 2016), which was produced by a second-generation impact on a Themis fragment (Nesvorný \& Morbidelli 2012). The vast majority of the studied Themis asteroids are classified as C- and B-types, directly associated with a carbonaceous composition similar to CM or CI meteorites (Fornasier et al. 2016). Several Themis family members appear to have the absorption band centred at $0.68-0.73 \mu \mathrm{m}$ indicating the presence of aqueously altered minerals (Fornasier et al. 2016). Further spectroscopic investigation in the range $2-4 \mu \mathrm{m}$ has revealed the presence of water ice on the family parent (24) Themis, due to the detection of the $3 \mu \mathrm{m}$ band (Rivkin \& Emery 2010; Campins et al. 2010).

However, a few of the observed Themis and Beagle family members have dubious or clearly different classifications.

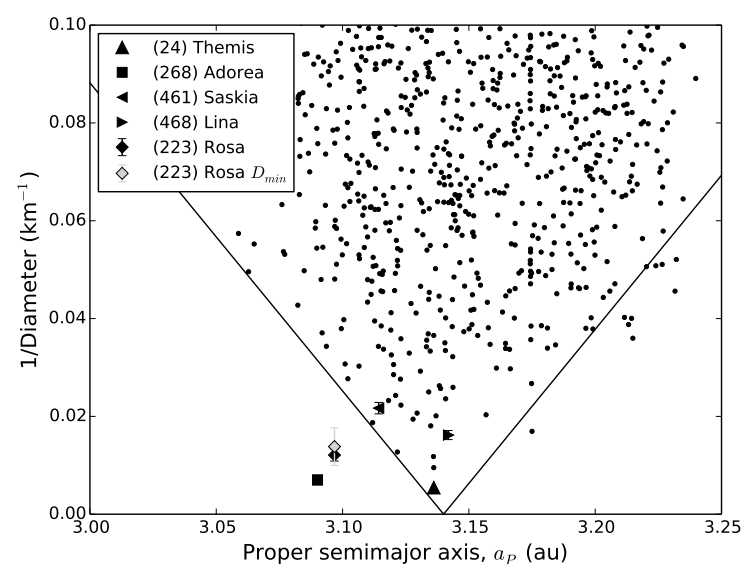

Fig. 3. V-shaped formation of the asteroid family Themis, and the positions (outside of the V) of asteroids (223) Rosa and (268) Adorea, currently considered Themis family members. Even if the smallest diameter is adopted for Rosa, the asteroid cannot be placed within the Themis $\mathrm{V}$ shape. The Themis family is truncated at $a_{p}=3.24 \mathrm{au}$, due to the presence of the nearby $2: 1$ mean motion resonance. The $\mathrm{V}$ shape is detected according to the procedure described in Appendix C.

The asteroids with dubious classifications are (2489) Suvorov and (3832) Shapiro, which are classified as C-complex in the Tholen taxonomy, while in Bus as X-complex (Lazzaro et al. 2004). In addition, asteroids that have a spectral classification that is not C or B are (268) Adorea, (461) Saskia, and (468) Lina, which are classified as X-complex asteroids in the Tholen, Bus, and/or Bus-DeMeo taxonomies (Lazzaro et al. 2004; Marsset et al. 2016). In this work we confirm that the mean spectrum of Rosa places it firmly in the Bus-DeMeo Xclass (or Tholen P-class), strengthening its interloper nature within the Themis family. Therefore, this is an indication that Rosa, Adorea, Saskia, and Lina could be interlopers in Themis. Here we should note that a spectral variation inside the same asteroid family is not always an indication of the existence of interlopers: Fornasier et al. (2007) showed that C and D/P-type asteroids may co-exist in the same families of Jupiter trojans. Moreover, Vernazza et al. (2021) proposed the idea that some dark objects may consist of different layers, namely a C-type core and D/P-type exterior layers; the breakup of such an object would create a spectrally inhomogeneous asteroid family.

Seeking further indications regarding the Themis membership from the asteroids above, apart from Rosa, (268) Adorea is also placed outside of the Themis V shape (see Fig. 3). Therefore, its location, along with its large diameter $(D=142.8 \pm$ $4.6 \mathrm{~km})$ and low albedo $\left(p_{V}=0.044 \pm 0.004\right)$, which is also marginally consistent with the Themis family, can additionally indicate a different origin for Adorea. On the other hand, the last two asteroids, (461) Saskia and (468) Lina, reside inside the Themis V shape. Saskia has a relatively small diameter, $D=46.1 \pm 2.5 \mathrm{~km}$, and $p_{V}=0.064 \pm 0.012$ that is very similar to the average value of the Themis family, while Lina is larger, with $D=61.8 \pm 3.5 \mathrm{~km}$, and darker, with $p_{V}=0.046 \pm 0.011$. Currently, we cannot firmly exclude them (especially Saskia) from the Themis family. Combining the old and new spectroscopic observations with the albedo of Rosa as well as its size and location (with respect to the Themis family's V shape), we favour the scenario that Rosa is not a Themis family member. Instead, we argue that it is an object that stayed intact, at least during its residence in the main belt.

The density of Rosa is typical for the carbonaceous C-complex and dark X-complex asteroids (Tholen's taxonomy 
P-types) (<2000 kg m${ }^{-3}$, Fienga et al. 2020; Carry 2012), but somewhat larger than the density that was estimated for large transneptunian objects (e.g., Grundy et al. 2019a,b) and two Jupiter trojans (Berthier et al. 2020; Brown et al. 2021). In any case, we caution that the density of Rosa is affected by a large uncertainty due to its currently uncertain mass $(\sim 50 \%$ uncertainty).

Starting from the Nice Model (Morbidelli et al. 2005), dynamical studies (Levison et al. 2009; Vokrouhlický et al. 2016) have demonstrated that at the initial stages of our Solar System evolution and during the giant planet instability, the population of primitive transneptunian objects was implanted in the inner Solar System and a part was captured in the main belt. This population, spectroscopically classified as D/P-types, should have formed beyond 15 au and should be similar to the Hildas (Wong et al. 2017) and the Jupiter trojans. These primitive objects are mainly associated with organics and opaque minerals consistent with low albedo values and featureless spectra. Therefore, Rosa, due to its low albedo, spectroscopic classification, and low density is likely representative of this population and is certainly attractive for further studies.

\section{Conclusions}

We presented a compilation of the known physical properties of asteroid (223) Rosa, which has been proposed as a potential main belt flyby target for ESA's JUICE space mission. We used literature spectra as well as our new spectroscopic observations to improve the spectral type of the target, in addition to the study of spectral variability. Due to its preliminary low density, its red and featureless reflectance spectrum (X- or P-type), and its very low albedo, Rosa could be a primitive object that was formed at the outer Solar System and captured in the main belt during the giant planet instability. The JUICE mission will be an unprecedented opportunity to encounter and study this class of bodies. A possible closer encounter with Rosa would be used for future comparative studies with the properties of the Jupiter trojans, which contain a large population of $\mathrm{P}$ types and are targets of NASA's ongoing LUCY space mission (Levison et al. 2021).

Acknowledgements. We thank the anonymous reviewer and the Editor for their constructive comments. CA, MD, JB, SF acknowledge support from ANR "ORIGINS" (ANR-18-CE31-0014). This activity has been partly realised under the ASI-INAF contract 2018-25-HH.0. CA and MD were Visiting Astronomers at the Infrared Telescope Facility, which is operated by the University of Hawaii under contract 80HQTR19D0030 with the National Aeronautics and Space Administration. This project has received funding from the European Union's Horizon 2020 research and innovation programme under grants agreement No 101004719 and No 730890. This work is partially based on observations collected at Copernico telescope (Asiago, Italy) of the Istituto Nazionale di Astrofisica (INAF) - Osservatorio Astronomico di Padova and with the Italian Telescopio Nazionale Galileo (TNG) operated on the island of La Palma, Spain, by the Centro Galileo Galilei of INAF. This work is based on data provided by the Minor Planet Physical Properties Catalogue (MP3C) of the Observatoire de la Côte d'Azur. CA and MB during the observations and preparation of this manuscript were visiting scientists at the Physics Department of the Aristotle University of Thessaloniki in Greece and thank Professors K. Tsiganis and G. Voyatzis for their hospitality.

\section{References}

Acton, C. H. 1996, Planet. Space Sci., 44, 65

Avdellidou, C., Delbo', M., \& Fienga, A. 2018, MNRAS, 475, 3419

Berthier, J., Descamps, P., Vachier, F., et al. 2020, Icarus, 352, 113990

Bida, T. A., Dunham, E. W., Massey, P., \& Roe, H. G. 2014, SPIE Conf. Ser., $9147,91472 \mathrm{~N}$

Bolin, B. T., Delbo, M., Morbidelli, A., \& Walsh, K. J. 2017, Icarus, 282, 290

Brož, M., Morbidelli, A., Bottke, W. F., et al. 2013, A\&A, 551, A117
Brown, M. E., Levison, H. F., Noll, K. S., et al. 2021, Planet. Sci. J., 2, 170 Bus, S. J., \& Binzel, R. P. 2002, Icarus, 158, 146

Campins, H., Hargrove, K., Pinilla-Alonso, N., et al. 2010, Nature, 464, 1320 Carry, B. 2012, P\&SS, 73, 98

Clark, B. E., Ziffer, J., Nesvorny, D., et al. 2010, J. Geophys. Res. (Planets), 115, E06005

Cushing, M. C., Vacca, W. D., \& Rayner, J. T. 2004, PASP, 116, 362

Delbo, M., Walsh, K., Bolin, B., Avdellidou, C., \& Morbidelli, A. 2017, Science, 357, 1026

Delbo, M., Avdellidou, C., \& Morbidelli, A. 2019, A\&A, 624, A69

de León, J., Pinilla-Alonso, N., Campins, H., Licandro, J., \& Marzo, G. A. 2012, Icarus, 218, 196

DeMeo, F. E., Binzel, R. P., Slivan, S. M., \& Bus, S. J. 2009, Icarus, 202, 160

Devogèle, M., Moskovitz, N., Thirouin, A., et al. 2019, AJ, 158, 196

Fienga, A., Deram, P., Viswanathan, V., et al. 2019, Notes Sci. Tech. Inst. Mec. Celeste, 109

Fienga, A., Avdellidou, C., \& Hanuš, J. 2020, MNRAS, 492, 589

Florczak, M., Lazzaro, D., Mothé-Diniz, T., Angeli, C. A., \& Betzler, A. S. 1999, A\&AS, 134, 463

Folkner, W. M., Williams, J. G., Boggs, D. H., Park, R. S., \& Kuchynka, P. 2014, Interplanet. Network Prog. Rep., 42-196, 1

Fornasier, S., Lazzarin, M., Barbieri, C., \& Barucci, M. A. 1999, A\&AS, 135, 65

Fornasier, S., Dotto, E., Hainaut, O., et al. 2007, Icarus, 190, 622

Fornasier, S., Clark, B. E., \& Dotto, E. 2011, Icarus, 214, 131

Fornasier, S., Lantz, C., Perna, D., et al. 2016, Icarus, 269, 1

Grasset, O., Dougherty, M. K., Coustenis, A., et al. 2013, Planet. Space Sci., 78,

Grundy, W. M., Noll, K. S., Buie, M. W., et al. 2019a, Icarus, 334, 30 Grundy, W. M., Noll, K. S., Roe, H. G., et al. 2019b, Icarus, 334, 62

Gulbis, A. A. S., Bus, S. J., Elliot, J. L., et al. 2011, PASP, 123, 461

Kaluna, H. M., Masiero, J. R., \& Meech, K. J. 2016, Icarus, 264, 62

Keller, H. U., Barbieri, C., Koschny, D., et al. 2010, Science, 327, 190

Küppers, M., Moissl, R., Vincent, J.-B., et al. 2012, Planet. Space Sci., 66, 71

Lamy, P. L., Faury, G., Jorda, L., Kaasalainen, M., \& Hviid, S. F. 2010, A\&A, 521, A19

Lazzaro, D., Angeli, C. A., Carvano, J. M., et al. 2004, Icarus, 172, 179

Levison, H. F., Bottke, W. F., Gounelle, M., et al. 2009, Nature, 460, 364

Levison, H. F., Olkin, C. B., Noll, K. S., et al. 2021, Planet. Sci. J., 2, 171

Magrin, S., La Forgia, F., Pajola, M., et al. 2012, Planet. Space Sci., 66, 43

Marsset, M., Vernazza, P., Birlan, M., et al. 2016, A\&A, 586, A15

Marsset, M., DeMeo, F. E., Binzel, R. P., et al. 2020, ApJS, 247, 73

Masiero, J. R., Mainzer, A. K., Grav, T., et al. 2011, ApJ, 741, 68

Masiero, J. R., Mainzer, A. K., Grav, T., et al. 2012, ApJ, 759, L8

Masiero, J. R., Grav, T., Mainzer, A. K., et al. 2014, ApJ, 791, 121

Masiero, J. R., Nugent, C., Mainzer, A. K., et al. 2017, AJ, 154, 168

Masiero, J. R., Mainzer, A. K., Bauer, J. M., et al. 2020, Planet. Sci. J., 1, 5

Massironi, M., Marchi, S., Pajola, M., et al. 2012, Planet. Space Sci., 66, 125

Morbidelli, A., Levison, H. F., Tsiganis, K., \& Gomes, R. 2005, Nature, 435, 462

Nesvorný, D., \& Morbidelli, A. 2012, AJ, 144, 117

Nesvorny, D., Broz, M., \& Carruba, V. 2015, Asteroids IV, 297

Nugent, C. R., Mainzer, A., Bauer, J., et al. 2016, AJ, 152, 63

Oliva, E. 2003, Mem. Soc. Astron. It., 74, 118

Palumbo, P., Jaumann, R., Cremonese, G., et al. 2014, 45th Annual Lunar and

Planetary Science Conference, 2094

Pilcher, F. 2012, Minor Planet Bull., 39, 171

Preusker, F., Scholten, F., Knollenberg, J., et al. 2012, Planet. Space Sci., 66, 54

Rayner, J. T., Toomey, D. W., Onaka, P. M., et al. 2003, PASP, 115, 362

Reddy, V., Emery, J. P., Gaffey, M. J., et al. 2009, Meteorit. Planet. Sci., 44, 1917

Rivkin, A. S., \& Emery, J. P. 2010, Nature, 464, 1322

Ryan, E. L., \& Woodward, C. E. 2010, AJ, 140, 933

Schulz, R., Sierks, H., Küppers, M., \& Accomazzo, A. 2012, Planet. Space Sci., 66,2

Sierks, H., Lamy, P., Barbieri, C., et al. 2011, Science, 334, 487

Spoto, F., Milani, A., \& Knežević, Z. 2015, Icarus, 257, 275

Tedesco, E. F., Noah, P. V., Noah, M., \& Price, S. D. 2002, AJ, 123, 1056

Tholen, D. J. 1989, in Asteroid Taxonomic Classifications, eds. R. P. Binzel, T Gehrels, \& M. S. Matthews, 1139

Thomas, N., Barbieri, C., Keller, H. U., et al. 2012, Planet. Space Sci., 66, 96

Usui, F., Kuroda, D., Müller, T. G., et al. 2011, PASJ, 63, 1117

Vernazza, P., Ferrais, M., Jorda, L., et al. 2021, A\&A, 654, A56

Vokrouhlický, D., Brož, M., Bottke, W. F., Nesvorný, D., \& Morbidelli, A. 2006, Icarus, 182, 118

Vokrouhlický, D., Bottke, W. F., \& Nesvorný, D. 2016, AJ, 152, 39

Wong, I., Brown, M. E., \& Emery, J. P. 2017, AJ, 154, 104

Zappalà, V., Cellino, A., Farinella, P., \& Knezevic, Z. 1990, AJ, 100, 2030

Zappalà, V., Bendjoya, P., Cellino, A., Farinella, P., \& Froeschlé, C. 1995, Icarus, 116,291

Zellner, B., Tholen, D. J., \& Tedesco, E. F. 1985, Icarus, 61, 355 


\section{Appendix A: Literature data}

Table A.1. Physical properties of (223) Rosa. Ref.: (a) Nugent et al. (2016), (b) Masiero et al. (2017), (c) Masiero et al. (2020), (d) Masiero et al. (2014), (e) Usui et al. (2011), (f) Masiero et al. (2011), (g) Tedesco et al. (2002), (h) Ryan \& Woodward (2010), (i) Masiero et al. (2012), (j) Fienga et al. (2019), (k) Folkner et al. (2014), (1) Pilcher (2012).

\begin{tabular}{llll}
\hline \hline Diameter $(\mathbf{D}, \mathbf{k m})$ & Albedo $(\mathbf{p V})$ & Method & $\mathbf{R}$. \\
\hline $72.33 \pm 20.18$ & $0.040 \pm 0.050$ & NEATM & $\mathrm{a}$ \\
$76.46 \pm 29.36$ & $0.035 \pm 0.023$ & NEATM & $\mathrm{b}$ \\
$79.68 \pm 33.85$ & $0.036 \pm 0.037$ & NEATM & $\mathrm{c}$ \\
$79.81 \pm 0.31$ & $0.040 \pm 0.010$ & NEATM & $\mathrm{d}$ \\
$80.93 \pm 1.46$ & $0.037 \pm 0.002$ & NEATM & $\mathrm{e}$ \\
$83.39 \pm 2.97$ & $0.034 \pm 0.005$ & NEATM & $\mathrm{f}$ \\
$86.05 \pm 26.43$ & $0.033 \pm 0.046$ & NEATM & $\mathrm{c}$ \\
$87.61 \pm 4.40$ & $0.031 \pm 0.003$ & STM & $\mathrm{g}$ \\
$88.50 \pm 3.79$ & $0.031 \pm 0.003$ & NEATM & $\mathrm{h}$ \\
$89.37 \pm 3.53$ & $0.030 \pm 0.002$ & STM & $\mathrm{h}$ \\
$90.43 \pm 20.33$ & $0.030 \pm 0.020$ & NEATM & $\mathrm{a}$ \\
$109.16 \pm 0.95$ & $0.020 \pm 0.003$ & NEATM & $\mathrm{i}$ \\
\hline Mass $(\mathbf{m}, \mathbf{k g})$ & & Method & $\mathbf{R}$. \\
\hline $5.979 \times 10^{17} \pm 2.971 \times 10^{17}$ & & Ephemeris & $\mathrm{j}$ \\
$9.350 \times 10^{17}$ & & Ephemeris & $\mathrm{k}$ \\
\hline Rotation period $\mathbf{( P , \mathbf { h } )}$ & & Method & $\mathbf{R}$. \\
\hline $20.283 \pm 0.002$ & & Lightcurve & 1 \\
\hline
\end{tabular}

Table A.2. Literature spectral and spectrophotometric data of Rosa, where $\alpha$ is the phase angle during the observations. Spectra were classified by the observers in Tholen (T), Bus (B), and Bus-DeMeo (BDM) taxonomies. (*) Eight Color Asteroid Survey (ECAS) data were classified by Tholen (1989). Ref.: (a) Zellner et al. (1985), (b) Lazzaro et al. (2004), (c) Fornasier et al. (2011), and (d) Marsset et al. (2016).

\begin{tabular}{lccll}
\hline \hline Date (UT) & $\alpha\left(^{\circ}\right)$ & Telescope & Class & Ref. \\
\hline $1980-03-17$ & 2.9 & Catalina & $\mathrm{X}^{T}$ & $(\mathrm{a})^{*}$ \\
$2001-01-29$ & 7.0 & La Silla & $\mathrm{X}^{B}, \mathrm{Xc}^{T}$ & $(\mathrm{~b})$ \\
$2004-11-20$ & 17.9 & TNG & $\mathrm{C}^{B}$ & $(\mathrm{c})$ \\
$2010-10-19$ & 1.0 & IRTF & $\mathrm{T}^{B D M}$ & $(\mathrm{~d})$ \\
\hline
\end{tabular}

\section{Appendix B: Spectroscopic observations}

We obtained NIR observations in August 2021 from the IRTF at Mauna Kea in Hawaii, using the SpeX (Rayner et al. 2003) instrument, and in October 2021 from the TNG, using the NICS instrument (Oliva 2003). SpeX was used with a slit $0.8 \times 15$ " in PRISM mode, covering wavelengths between 0.7 and $2.5 \mu \mathrm{m}$ with a spectral resolution of 200 in a single configuration. MORIS (Gulbis et al. 2011) was used as the IRTF auto-guider camera during the acquisition of the spectral data. NICS was used with a 2" slit and the AMICI grism, covering wavelengths between 0.8 and $2.5 \mu \mathrm{m}$. In the NIR we used the commonly adopted ABBA observation procedure which consists in alternating our targets between two positions along the slit (A and $\mathrm{B})$, thus allowing us to remove the background contribution.

The VIS spectra were observed in August and October 2021 at the Copernico Telescope in Asiago, using the Asiago

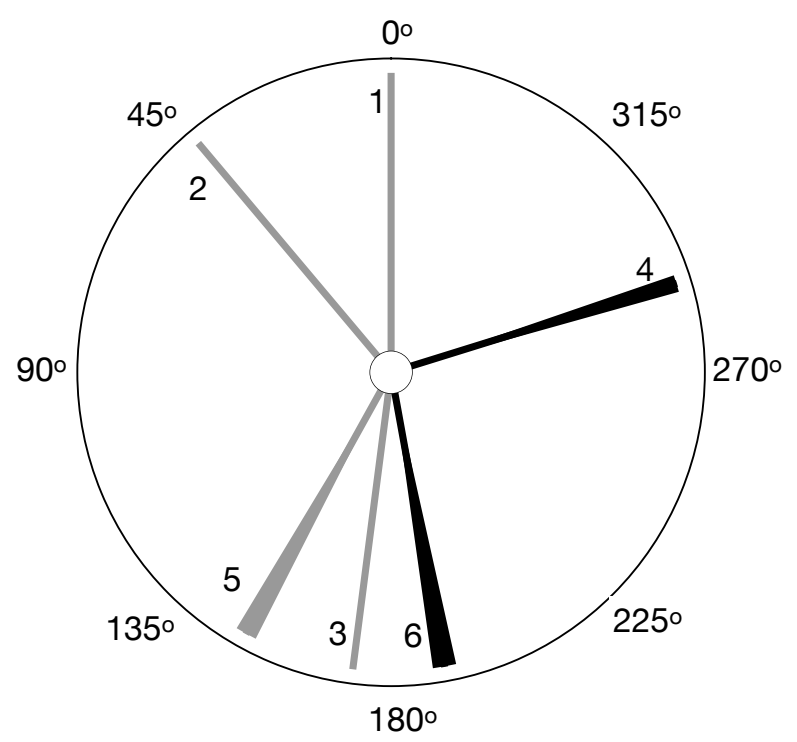

Fig. B.1. Rotational phase plot for each observation. Like a dial in a meter, each line represents the rotational phase of the asteroid at the time of each observation (see Table 1 for the epoch of the observations). The grey dials correspond to observations in the NIR, while black ones to observations in the VIS. The width of the bars is related to the accuracy of the knowledge of the phase due to the propagation of the uncertainty in the rotational period. The phase is arbitrarily set to zero at the epoch of the first observation.

Faint Object Spectrograph (AFOSC), and at the Lowell Discovery Telescope (LDT) in Arizona, using the DeVeny optical spectrograph (Bida et al. 2014). AFOSC was used with the VPH\#6 grism covering the $0.4-1.0 \mu \mathrm{m}$ range and the 4.22 arcsec slit resulting in a spectral resolution of $0.295 \mathrm{~nm} \mathrm{pix}^{-1}$. DeVeny was used with the R150 grating providing a dispersion of $0.43 \mathrm{~nm} \mathrm{pix}{ }^{-1}$ covering a spectral range between 0.32 and $1 \mu \mathrm{m}$. In the NIR we collected data with the slit oriented to the parallactic angle so that any atmospheric dispersion is along the slit and the light loss is minimised. It is also important to measure the correct spectral shape because the spectral slope variation is much reduced when both the target and standard star are observed at the parallactic angle. ${ }^{6}$ In the VIS observations from Asiago the rotator was not used because the 4.22 arcsec slit was large enough to compensate for differential refraction.

The data were reduced with different programs according to the instrument used: SpeX observations were reduced using the interactive data language (IDL)-based spectral reduction tool Spextool (v4.1) (Cushing et al. 2004); AFOSC and TNG data were analysed using the ESO-MIDAS with standard reduction methods (Fornasier et al. 1999); DeVeny data were reduced using standard reduction techniques for spectroscopy, as detailed in Devogèle et al. (2019). In all cases we performed flat fielding and wavelength calibration. For both NIR and VIS observations we observed a trusted solar analogue star at similar airmass with which we divided the asteroid spectrum in order to derive the reflectance: $R(\lambda)=\frac{A(\lambda)}{S_{T}(\lambda)}$. During the NIR observations, following the established observational methods (Reddy et al. 2009), we observed an additional local G2 star when it was possible to be found within 300 arcmin maximum distance from our target. This was used in order to perform one more intermediate step in the data reduction: we first corrected the asteroid spectrum for the telluric absorption and then divided with the trusted solar

6 http://irtfweb.ifa.hawaii.edu/ spex/observer/ 

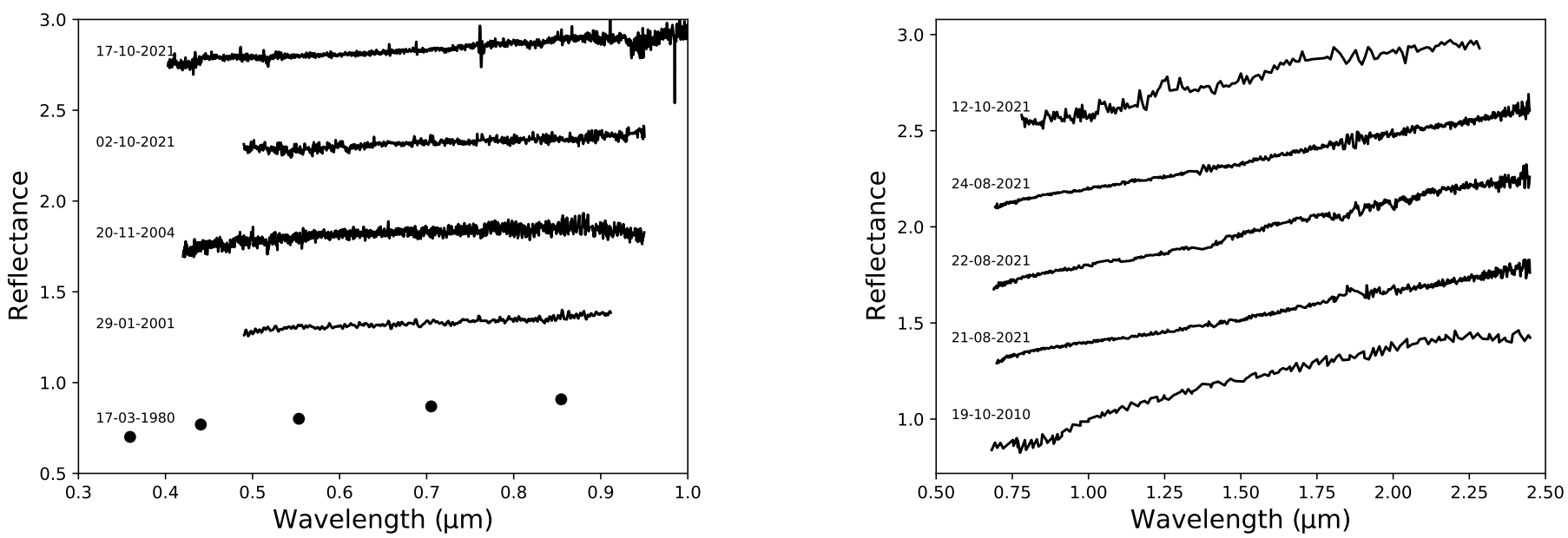

Fig. B.2. Compilation of literature and new VIS (left) and NIR (right) spectra of Rosa, presented in chronological order. Observations obtained on 21-08-2021, 22-08-2021, 24-08-2021, 2-10-2021, 12-10-2021, and 17-10-2021 are from this study, while observations on 17-03-1980 are from Zellner et al. (1985), on 29-01-2001 are from Lazzaro et al. (2004), on 20-11-2004 are from Fornasier et al. (2011), and on 19-10-2010 are from Marsset et al. (2016).

analogues (Table 1). Our new spectra together with the literature spectra are presented in Fig. B.2.

\section{Appendix C: Themis family}

We used the method of Bolin et al. (2017) with the specific implementation of Delbo et al. (2017) to determine the borders and the centre of the Yarkovsky V shape of the Themis family. This method searches for $\mathrm{V}$ shapes of unknown borders and the vertices in an asteroid population, in the $2 \mathrm{D}$ space of parameters representing the centre of the family $a_{C}$ and the slope $K$ of the sides of the $\mathrm{V}$ shape. For each value of $K$, the method counts the number of asteroids in the section of the $(a, 1 / D)$ space just above and just below the lines of $1 / D=K\left|a-a_{c}\right|$ :

$N_{a}=\sum_{i=0}^{N} w_{i}$; with $w_{i}=1$ if $K\left|a_{i}-a_{c}\right| \leq 1 / D_{i}<K\left|a_{i}-a_{c}\right|+K a_{w} ;$ $w_{i}=0$ otherwise,

$N_{b}=\sum_{i=0}^{N} w_{i} ;$ with $w_{i}=1$ if $K\left|a_{i}-a_{c}\right|-K a_{w} \leq 1 / D_{i}<K\left|a_{i}-a_{c}\right|$ $w_{i}=0$ otherwise.

Here $i$ indexes each asteroid of the sample, $a_{w}$ is the width of the section projected to the $a$-axis, $N_{\text {in }}$ and $N_{\text {out }}$ are respectively the numbers of asteroids in the section above and below the sides of the $\mathrm{V}$ shape as a function of $a_{c}$ and $K$. We chose $a_{w}=0.03$ from Delbo et al. (2017). This process builds a map of the quantity

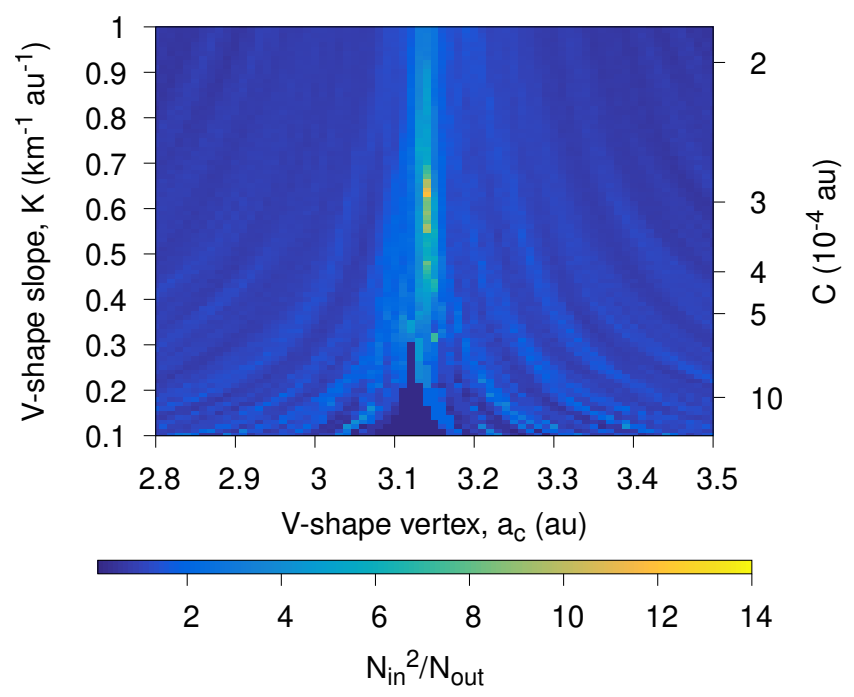

Fig. C.1. Value of $N_{\text {in }}^{2} / N_{\text {out }}$ plotted as a function of the slope $(K)$ and semimajor axis of the vertex of the $\mathrm{V}$ shape $\left(a_{c}\right)$. The value of the parameter $C$, which measures the width of a $\mathrm{V}$ shape in the $(a, H)$ space, where $H$ is the absolute magnitude (Vokrouhlický et al. 2006), is calculated from $C=1 / K \sqrt{p_{V}} / 1329$ using the average geometric visible albedo of the Themis family (see text).

$N_{\text {in }}^{2} / N_{\text {out }}$ (Fig. C.1) as a function of $a_{c}$ and $K$. The maximum of $N_{\text {in }}^{2} / N_{\text {out }}$ indicates the slope $\left.(K=0.63) \mathrm{au}^{-1} \mathrm{~km}^{-1}\right)$ and centre $\left(a_{c}=3.14 \mathrm{au}\right)$ of the $\mathrm{V}$ shape. 